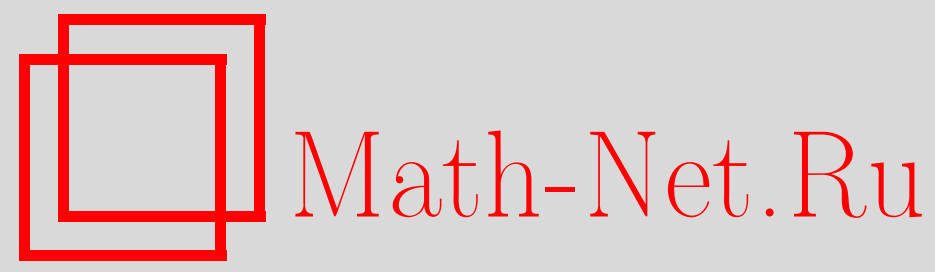

M. V. Nikolaev, O сложности двупараметрической задачи дискретного логарифмирования в конечной циклической группе с эффективным автоморфизмом, Матем. вопр. криптогр., 2015, том 6, выпуск $2,45-57$

DOI: https://doi.org/10.4213/mvk144

Использование Общероссийского математического портала Math-Net.Ru подразумевает, что вы прочитали и согласны с пользовательским соглашением http://www . mathnet.ru/rus/agreement

Параметры загрузки:

IP : 3.81 .55 .215

26 апреля 2023 г., 13:24:41 


\title{
On the complexity of two-dimensional discrete logarithm problem in a finite cyclic group with efficient automorphism
}

\author{
M. V. Nikolaev \\ Lomonosov Moscow State University, Moscow
}

Получено 16.IX.2014

Two-dimensional discrete logarithm problem in a finite additive group $G$ consists in solving the equation $Q=n_{1} P_{1}+n_{2} P_{2}$ with respect to $n_{1}, n_{2}$ for specified $P_{1}, P_{2}, Q \in G, 0<N_{1}, N_{2}<\sqrt{|G|}$ such that there exists solution with $\left|n_{1}\right| \leq$ $N_{1},\left|n_{2}\right| \leq N_{2}$. In 2004, Gaudry and Schost proposed an algorithm to solve this problem with average complexity $(c+o(1)) \sqrt{N}$ of group operations in $G$ where $c \approx 2.43, N=4 N_{1} N_{2}, N \rightarrow \infty$. In 2009, Galbraith and Ruprai improved this algorithm to obtain $c \approx 2.36$. We show that the constant $c$ may be reduced if the group $G$ has an automorphism computable faster than the group operation.

Key words: two-dimensional discrete logarithm problem, Gaudry-Schost algorithm, elliptic curve, efficient automorphism 


\section{О сложности двупараметрической задачи дискретного логарифмирова- ния в конечной циклической группе с эффективным автоморфизмом}

\section{М. В. Николаев}

Московский государственный университет имени М. В. Ломоносова, Москва

Аннотация. Двупараметрическая задача дискретного логарифмирования в конечной аддитивной группе $G$ заключается в решении уравнения $Q=n_{1} P_{1}+n_{2} P_{2}$ относительно $n_{1}, n_{2}$ для заданных $P_{1}, P_{2}, Q \in G, 0<N_{1}, N_{2}<\sqrt{|G|}$ в случае, когда существует такое решение $\left(n_{1}, n_{2}\right)$, что $\left|n_{1}\right| \leq N_{1},\left|n_{2}\right| \leq N_{2}$. В 2004 г. Gaudry и Schost предложили алгоритм решения этой задачи со сложностью порядка $(c+o(1)) \sqrt{N}$ операций в группе $G$, где $c \approx 2.43, N=4 N_{1} N_{2}, N \rightarrow \infty$. В 2009 г. Galbraith и Ruprai улучшили этот алгоритм, получив $c \approx 2.36$. Мы показываем, что если в группе $G$ существует автоморфизм, вычисляемый быстрее групповой операции, то можно улучшить эти алгоритмы и уменьшить оценку $c$.

Ключевые слова: двупараметрическая задача дискретного логарифмирования, алгоритм Годри-Шоста, эллиптическая кривая, эффективный автоморфизм

Citation: Mathematical Aspects of Cryptography, 2015, vol. 6, no. 2, pp. $45-57$ (Russian).

(C) 2015 M. V. Nikolaev 


\section{Introduction}

Definition 1. Discrete logarithm problem.

Given: group $G=\langle P\rangle, Q \in G$.

To find: $n \in\{0, \ldots,|G|-1\}$ such that $Q=n P$.

Definition 2. Two-dimensional discrete logarithm problem.

Given: group $G ; P_{1}, P_{2}, Q \in G, N_{1}, N_{2} \in \mathbb{N}, Q=n_{1} P_{1}+n_{2} P_{2}$ for some (unknown) $n_{1} \in\left\{-N_{1}, \ldots, N_{1}\right\}, n_{2} \in\left\{-N_{2}, \ldots, N_{2}\right\}$.

To find: $n_{1}^{\prime}, n_{2}^{\prime} \in \mathbb{Z}$ such that $Q=n_{1}^{\prime} P_{1}+n_{2}^{\prime} P_{2}$.

In the general case the most effective algorithm of solving two-dimensional discrete logarithm problem is the Gaudry-Schost algorithm proposed in 2004 in [4]. Main idea of this algorithm may be described as follows. First, select so-called «tame» and «wild» sets:

$$
\begin{gathered}
T=\left\{-N_{1}, \ldots, N_{1}\right\} \times\left\{-N_{2}, \ldots, N_{2}\right\}, \\
W=\left\{-N_{1}+n_{1}, \ldots, N_{1}+n_{1}\right\} \times\left\{-N_{2}+n_{2}, \ldots, N_{2}+n_{2}\right\} .
\end{gathered}
$$

Second, we calculate in parallel two pseudorandom sequences

$$
\begin{gathered}
x_{i} P_{1}+y_{i} P_{2}, \quad\left(x_{i}, y_{i}\right) \in T, i=1,2, \ldots, \\
Q+z_{j} P_{1}+w_{j} P_{2}, \quad\left(n_{1}+z_{j}, n_{2}+w_{j}\right) \in W, j=1,2, \ldots,
\end{gathered}
$$

until we obtain two identical elements:

$$
x_{k} P_{1}+y_{k} P_{2}=Q+z_{l} P_{1}+w_{l} P_{2},
$$

from which we find $n_{1}^{\prime}=x_{k}-z_{l}, n_{2}^{\prime}=y_{k}-w_{l}$.

The average complexity of the Gaudry-Schost algorithm and its various modifications (measured by the number of group operations in $G$ ), is proportional to the mean number of elements of sequences (1), (2) calculated before obtaining elements satisfying (3); we assume that the values $\left(n_{1}, n_{2}\right),\left(x_{i}, y_{i}\right)$ and $\left(z_{j}, w_{j}\right)$ are chosen from the corresponding sets at random independently and with equal probabilities. This average value is determined using the following result due to Galbraith and Holmes, which is a generalization of the birthday paradox. 
Theorem 1 ([1, Theorem 1]). Let the following conditions are fulfilled.

1. In a random sequence of balls of $C>1$ different colors $k$-th ball with probability $r_{k, c}$ is of the color $c$ (independently of previously appeared balls). For each $c=1, \ldots, C$ there exists $p_{c}=\lim _{n \rightarrow \infty} n^{-1} \sum_{k=1}^{n} r_{k, c}$ and $p_{1} \geq$ $p_{2} \geq \cdots \geq p_{C}>0$. Let $b_{n, c}=p_{c}-n^{-1} \sum_{k=1}^{n} r_{k, c}$; and there exists a constant $K$ such that $\left|b_{n, c}\right| \leq K / n$ for any $c=1, \ldots, C, n>1$.

2. There exist $N^{\prime} \in \mathbb{N}$ different boxes. If $k$-th ball has a color $c$, then it falls into the $i$-th box with probability $q_{c, i}\left(N^{\prime}\right)$ independently of previous balls. There exists an absolute constant $d>0$ such that for all $c=1, \ldots, C$ and $i=1, \ldots, N^{\prime}$

$$
0 \leq q_{c, i} \leq d / N^{\prime}
$$

There are constants $\alpha, \mu>0$ such that $\mid\left\{i \in\left\{1, \ldots, N^{\prime}\right\}: q_{1, i}, q_{2, i} \geq\right.$ $\left.\mu / N^{\prime}\right\} \mid \geq \alpha N^{\prime}$

Then the number $Z_{N^{\prime}}$ of balls allocated before the first occurrence of two balls of different colors in the same urn has the mean

$$
\mathbf{M}\left(Z_{N^{\prime}}\right)=\sqrt{\frac{\pi}{2 A_{N^{\prime}}}}+O\left(N^{\prime 1 / 4}\right),
$$

where

$$
A_{N^{\prime}}=\sum_{c=1}^{C} p_{c}\left(\sum_{c^{\prime}=1, c \neq c^{\prime}}^{C} p_{c^{\prime}}\left(\sum_{i=1}^{N^{\prime}} q_{c, i} q_{c^{\prime}, i}\right)\right)
$$

and constant in $O$ depends on $C, p_{c}, d, K, \alpha, \mu$, but does not depend on $N^{\prime}$ and $q_{c, i}$.

The average complexity of the Gaudry-Schost algorithm was calculated by Galbraith and Ruprai in [2] and equals to $(2.43+o(1)) \sqrt{N}$, where $N=4 N_{1} N_{2}$, $o(1) \rightarrow 0$ for $N_{1}, N_{2} \rightarrow \infty$. In the same paper an improved version of the algorithm was proposed with complexity $(2.36+o(1)) \sqrt{N}$.

Suppose now that the group $G$ is cyclic of prime order and has an automorphism $\varphi$ such that set of elements $\left\{\varphi(P), \ldots, \varphi^{\operatorname{ord}(\varphi)-1}(P)\right\}$ may be computed significantly faster than the group operation for any $P \in G$, and it is easy to calculate $\lambda \in\{1, \ldots, n-1\}$ such that $\varphi$ is equivalent to the multiplication by $\lambda$ in group $G$. Then the group $G$ decomposes into disjoint equivalence classes (orbits) under the action of $\langle\varphi\rangle$, and analogously to the solution of the classical discrete logarithm problem in [6] we may speed up the algorithm if we stop the search after appearance of elements of sequences (1) and (2) belonging to the 
same equivalence class. Indeed, in this case instead of equality (3) we obtain the equality

$$
\varphi^{s}\left(x_{k} P_{1}+y_{k} P_{2}\right)=Q+z_{l} P_{1}+w_{l} P_{2}
$$

for some $s$, leading to the representation

$$
Q=\left(\lambda^{s} x_{k}-z_{l}\right) P_{1}+\left(\lambda^{s} y_{k}-w_{l}\right) P_{2},
$$

i. e. $n_{1}^{\prime}=\lambda^{s} x_{k}-z_{l}, n_{2}^{\prime}=\lambda^{s} y_{k}-w_{l}$.

For example, subgroups of elliptic curves over prime finite field $G F(p)$ are groups with such efficient automorphisms.

1. Curve given by the equation $y^{2}=x^{3}+A x+B$ over prime finite field with $p>3$ elements obviously has an automorphism of order 2 with $\lambda=-1$ (the operation of inversion element $\varphi(x, y)=(x,-y))$. If $P_{2}=\varphi\left(P_{1}\right)$, then

$$
\varphi\left(a P_{1}+b P_{2}\right)=a\left(\lambda P_{1}\right)+b\left(\lambda^{2}\right) P_{1}=b P_{1}+a P_{2},
$$

the equivalence class of the point $a P_{1}+b P_{2}$ under the action of $\langle\varphi\rangle$ includes $a P_{1}+b P_{2}$ and $\varphi\left(a P_{1}+b P_{2}\right)$. Each such equivalence class corresponds to the set (class) of pairs

$$
C(a, b)=\{(a, b),(b, a)\} .
$$

In Liu [5] for this case appropriate modifications of the Gaudry-Schost algorithm were suggested with asymptotic complexity (if $N_{1}=N_{2}, N \rightarrow \infty$ ) $(1.45+o(1)) \sqrt{N}$. To obtain this result, Liu uses «tame» set

$$
\begin{aligned}
T=\{(a, b),(-a,-b)\}: & \\
& \left.-\frac{17}{20} N_{1} \leq a \leq \frac{17}{20} N_{1},-\frac{17}{20} N_{1} \leq b \leq \frac{17}{20} N_{1}\right\} .
\end{aligned}
$$

as well as «wild» set

$$
\begin{aligned}
W=\left\{\left\{\left(n_{1}+a, n_{2}+b\right),\left(-n_{1}-a,\right.\right.\right. & \left.\left.-n_{2}-b\right)\right\}: \\
& \left.-\frac{N_{1}}{2} \leq a \leq \frac{N_{1}}{2},-\frac{N_{1}}{2} \leq b \leq \frac{N_{1}}{2}\right\}
\end{aligned}
$$

Under the action of automorphism $\varphi$ the set of class representatives for each $C(a, b) \in T$ is equal to

$$
\widetilde{T}=\left\{(a, b):-\frac{17}{20} N_{1} \leq a \leq \frac{17}{20} N_{1},-a \leq b \leq \frac{17}{20} N_{1}\right\}
$$

Fig. 1 displays «wild» set, set $T_{0}$ (union of the elements of the set $T$ ), as well as the intersection $U=\widetilde{W} \cap \widetilde{T}$, where

$$
\widetilde{W}=\left\{\left(n_{1}+a, n_{2}+b\right):-\frac{N_{1}}{2} \leq a \leq \frac{N_{1}}{2},-\frac{N_{1}}{2} \leq b \leq \frac{N_{1}}{2}\right\} .
$$




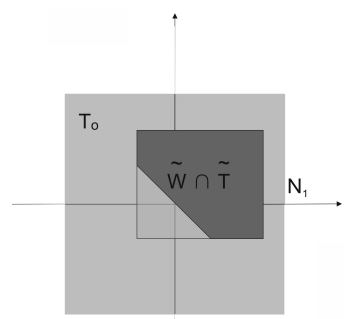

Fig. 1.

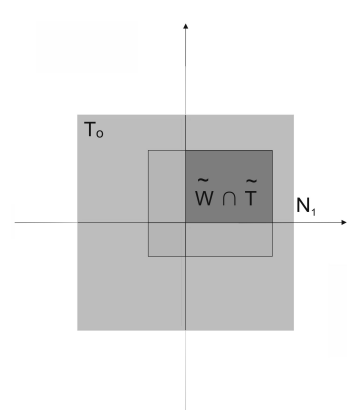

Fig. 2.

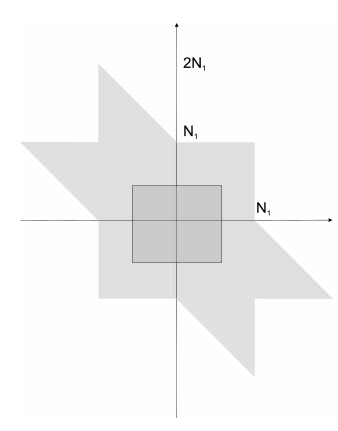

Fig. 3.

2. Curve given by the equation $y^{2}=x^{3}+A x$ with $p \equiv 1 \bmod 4$ has an automorphism of order 4: $\varphi(x, y)=(-x, \alpha y)$, where $\alpha$ is the element of order 4 modulo $p, \lambda$ is the root of equation $\lambda^{2} \equiv-1(\bmod n)$. If $P_{2}=\varphi\left(P_{1}\right)$ then

$$
\varphi\left(a P_{1}+b P_{2}\right)=a\left(\lambda P_{1}\right)+b\left(\lambda^{2}\right) P_{1}=-b P_{1}+a P_{2},
$$

whence

$$
\begin{gathered}
\varphi^{2}\left(a P_{1}+b P_{2}\right)=-a P_{1}-b P_{2}, \\
\varphi^{3}\left(a P_{1}+b P_{2}\right)=b P_{1}-a P_{2},
\end{gathered}
$$

the equivalence class of the point $a P_{1}+b P_{2}$ under the action of $\langle\varphi\rangle$ includes 4 points. Each such equivalence class corresponds to the set (class) of pairs

$$
C(a, b)=\{(a, b),(-a, b),(-a,-b),(a,-b)\} .
$$

In Liu [5] for this case some appropriate modifications of the GaudrySchost algorithm were suggested with asymptotic complexity (if $N_{1}=N_{2}$ ) $(1.0255+o(1)) \sqrt{N}$. To obtain this result, Liu uses «tame» set

$$
\begin{aligned}
T=\{ & \{(a, b),(-a, b),(-a,-b),(a,-b)\}: \\
& \left.-(1-\tau) N_{1} \leq a \leq(1-\tau) N_{1},-(1-\tau) N_{1} \leq b \leq(1-\tau) N_{1}\right\},
\end{aligned}
$$

where $\tau=0.1467$, as well as «wild» set

$$
\begin{aligned}
W=\left\{\left\{\left(n_{1}+a, n_{2}+b\right),\left(-n_{1}-a,\right.\right.\right. & \left.\left.-n_{2}-b\right)\right\}: \\
& \left.-\frac{N_{1}}{2} \leq a \leq \frac{N_{1}}{2},-\frac{N_{1}}{2} \leq b \leq \frac{N_{1}}{2}\right\} .
\end{aligned}
$$


Under the action of automorphism $\varphi$ the set of class representatives for each $C(a, b) \in T$ is equal to

$$
\widetilde{T}=\left\{(a, b): 0 \leq a \leq(1-\tau) N_{1}, 0 \leq b \leq(1-\tau) N_{1}\right\} .
$$

Fig. 2 displays «wild» set, set $T_{0}$ (union of the elements of the set $T$ ), as well as the intersection $U=\widetilde{W} \cap \widetilde{T}$, where

$$
\widetilde{W}=\left\{\left(n_{1}+a, n_{2}+b\right):-\frac{N_{1}}{2} \leq a \leq \frac{N_{1}}{2},-\frac{N_{1}}{2} \leq b \leq \frac{N_{1}}{2}\right\} .
$$

3. Curve $E$ over $G F(p)$ given by the equation $y^{2}=x^{3}+B$ with $p \equiv 1(\bmod 3)$ has an automorphism $\varphi$ of order $6, \varphi(x, y)=(\beta x,-y)$ where $\beta \neq 1$ is the cube root of 1 modulo $p, \lambda$ is the root of equation $\lambda^{2}-\lambda+1 \equiv 0(\bmod n)$. If $P_{2}=\varphi\left(P_{1}\right)$ then

$$
\varphi\left(a P_{1}+b P_{2}\right)=a\left(\lambda P_{1}\right)+b(\lambda-1) P_{1}=-b P_{1}+(a+b) P_{2},
$$

whence

$$
\begin{gathered}
\varphi^{2}\left(a P_{1}+b P_{2}\right)=-(a+b) P_{1}+a P_{2}, \\
\varphi^{3}\left(a P_{1}+b P_{2}\right)=-a P_{1}-b P_{2}, \\
\varphi^{4}\left(a P_{1}+b P_{2}\right)=b P_{1}-(a+b) P_{2}, \\
\varphi^{5}\left(a P_{1}+b P_{2}\right)=(a+b) P_{1}-a P_{2},
\end{gathered}
$$

i. e. the equivalence class of the point $a P_{1}+b P_{2}$ under the action of $\langle\varphi\rangle$ includes six points. Each such equivalence class corresponds to the set (class) of pairs

$$
\begin{aligned}
C(a, b)=\{(a, b),(-b, a+b), & (-(a+b), a), \\
& (-a,-b),(b,-(a+b)),(a+b,-a)\} .
\end{aligned}
$$

In the paper [6] for this case appropriate modifications of the Gaudry-Schost algorithm were suggested with asymptotic complexity $(0.9781+o(1)) \sqrt{N}$ (if $N \rightarrow \infty, N_{1}=N_{2}$ ). This result was obtained with «tame» set

$$
T=\left\{C(a, b):-N_{1} \leq a \leq N_{1},-N_{1} \leq b \leq N_{1}\right\}
$$

and «wild» set

$$
W=\left\{C\left(n_{1}+a, n_{2}+b\right):-\frac{N_{1}}{2} \leq a \leq \frac{N_{1}}{2},-\frac{N_{1}}{2} \leq b \leq \frac{N_{1}}{2}\right\} .
$$

Under the action of automorphism $\varphi$ the set of class representatives for each $C(a, b) \in T$ is equal

$$
\widetilde{T}=\left\{(a, b): 0 \leq a \leq N_{1}, 0 \leq b \leq N_{1}\right\} .
$$

Fig. 3 displays «wild» set and set $T_{0}$ (union of the elements of the set $T$ ). 


\section{New results}

We may vary the size of «tame» and «wild» sets to optimize the GaudrySchost algorithm. In order to determine the effect of resizing and reshaping these sets on a constant in the leading term in the average complexity, Python script was developed (package «shapely» was used). Three cases mentioned above were considered: similar «wild» and «tame» sets used for each of them, but the sizes of the «wild» sets were varied. Theorem 1 was used for calculations of $A_{N^{\prime}}$. The calculations show that the asymptotic estimates of the average complexity of the algorithm in all three cases decrease with decreasing intersection $U=\widetilde{W_{\tau}} \cap T_{0}$. Let give a rigorous proof.

Theorem 2. Let $G$ be a subgroup of prime order $n$ of the elliptic curve $E$ defined over a finite field $G F(p)$ by equation $y^{2}=x^{3}+B, p \equiv 1(\bmod 3) ; \varphi$ be an automorphism of group $G, \varphi(x, y)=(\beta x,-y)$, where $\beta \neq 1$ is the cube root of 1 modulo $p ; \lambda$ is the root of the equation $\lambda^{2}-\lambda+1 \equiv 0(\bmod n)$ such that $\varphi(x, y)=\lambda(x, y)$. Then for any $\varepsilon>0$ there exists an algorithm for the solution of the two-dimensional discrete logarithm problem in the group $G$ with average complexity $(1+\varepsilon) \sqrt{\frac{\pi N}{4}}+O\left(N^{\frac{1}{4}}\right)$ group operations (if $N_{1}=N_{2}, P_{2}=\varphi\left(P_{1}\right)$ and random equiprobable choice of $\left.\left(n_{1}, n_{2}\right)\right)$, where $N=4 N_{1} N_{2}, N \rightarrow \infty$.

\section{Proof.}

Define «tame» set $T$ and set $\widetilde{T}$ of class representatives of the elements of $T$ by

$$
T=\left\{C(a, b):-N_{1} \leq a \leq N_{1},-N_{1} \leq b \leq N_{1}\right\}
$$

and

$$
\widetilde{T}=\left\{0, \ldots, N_{1}\right\} \times\left\{0, \ldots, N_{1}\right\}
$$

(in the articles [3,5] such set was called a fundamental domain of «tame» set). Let $T_{0}$ be the union of the classes of the set $T$. Let parameter $\tau$ be a compression ratio of «wild» set $W$. Then

$$
W_{\tau}=\left\{C\left(n_{1}+a, n_{2}+b\right):-\frac{k N_{1}}{2} \leq a \leq \frac{k N_{1}}{2},-\frac{k N_{1}}{2} \leq b \leq \frac{k N_{1}}{2}\right\}
$$

and

$$
\widetilde{W}_{\tau}=\left\{-\frac{k N_{1}}{2}+n_{1}, \ldots, \frac{k N_{1}}{2}+n_{1}\right\} \times\left\{-\frac{k N_{1}}{2}+n_{2}, \ldots, \frac{k N_{1}}{2}+n_{2}\right\} .
$$

As in the Gaudry-Schost algorithm, we will calculate in the parallel the sequences of points

$$
\begin{gathered}
x_{i} P_{1}+y_{i} P_{2},\left(x_{i}, y_{i}\right) \in \widetilde{T}, i=1,2, \ldots, \\
Q+z_{j} P_{1}+w_{j} P_{2},\left(z_{j}, w_{j}\right) \in \widetilde{W}_{\tau}, j=1,2, \ldots
\end{gathered}
$$


Having obtained two points from different sequences in the same equivalence class we may find the solution of the problem as in Section 1. (This assumes that the values of $\left(x_{i}, y_{i}\right)$ and $\left(z_{j}, w_{j}\right)$ are chosen randomly, equiprobably and independently from respective sets.)

Obviously, the average complexity measured in group operations does not exceed the expectation of the total number $Z_{N^{\prime}}$ of values $\left(x_{i}, y_{i}\right)$ and $\left(n_{1}+z_{j}, n_{2}+\right.$ $\left.+w_{j}\right)$ chosen before the appearance of $\left(x_{k}, y_{k}\right)$ and $\left(n_{1}+z_{l}, n_{2}+w_{l}\right)$ such that $C\left(x_{k}, y_{k}\right)=C\left(n_{1}+z_{l}, n_{2}+w_{l}\right)$. Let us calculate the conditional expectation $\mathbf{M}\left(Z_{N^{\prime}} \mid\left(n_{1}, n_{2}\right)\right)$ of random variable $Z_{N^{\prime}}$ given $n_{1}, n_{2}$ by means of Theorem 1 as it was done in [6]. In this case $C=2$ : balls of color 1 are the elements of the set $\widetilde{T}$, and balls of color 2 are the elements of the set $\widetilde{W}_{\tau}$. The sequences (5) and (6) being calculated in parallel, we may assume that $r_{k, 1}=r_{k, 2}=\frac{1}{2}$ for all $k=1,2, \ldots$, that is $p_{1}=p_{2}=\frac{1}{2}$. Now the set of boxes is $T \cup W_{\tau}$, and the ball $(a, b)$ is allocated into the box $C(a, b)$. It is clear that $N^{\prime}=O(N)$. In order to simplify the formulas we shall omit the additive terms of the form $o(N)$ as $N \rightarrow \infty$. Then we have:

$$
q_{1, i}= \begin{cases}\frac{4}{N}, & \text { if } i \in T \\ 0, & \text { otherwise. }\end{cases}
$$

In accordance to the last equation and the theorem 1 we are interested in values of $q_{2, i}$ for $i \in T \cap W_{\tau}$ only. Each class $C(a, b)$ contains no more than 6 elements, so $T \cap W_{\tau}$ is divided into six disjoint subsets $U_{j}, j=1, \ldots, 6$, such that each class of $U_{j}$ contains exactly $j$ elements of $\widetilde{W}_{\tau}$, i. e.

$$
q_{2, i}=\frac{j}{\left|\widetilde{W}_{\tau}\right|}, i \in U_{j}
$$

From the last two equations we obtain

$$
A_{N^{\prime}}=2 \cdot \frac{1}{2} \cdot \frac{1}{2} \cdot \frac{4}{N} \cdot \frac{1}{\left|\widetilde{W}_{\tau}\right|} \cdot \sum_{j=1}^{6} j \cdot\left|U_{j}\right|=\frac{2|U|}{N\left|\widetilde{W}_{\tau}\right|}
$$

where $U=\widetilde{W}_{\tau} \cap T_{0}$, and by theorem 1

$$
\mathbf{M}\left(Z_{N^{\prime}} \mid\left(n_{1}, n_{2}\right)\right)=\sqrt{\frac{\pi}{2 A_{N^{\prime}}}}+O\left(N^{\prime \frac{1}{4}}\right) .
$$

Following the articles $[3,5]$, we put $n_{1}=x N_{1}, n_{2}=y N_{1},|x|,|y| \leq 1$, and consider different cases of the arrangement of the set $U$ depending on the values $x$ and $y$. 
1) $\left(n_{1}, n_{2}\right) \in B_{1}=\left\{\left(x N_{1}, y N_{1}\right):|x| \leq 1-\frac{1}{2} \tau,|y| \leq 1-\frac{1}{2} \tau\right\}$ (see. Fig.4). Probability of the event $\left(n_{1}, n_{2}\right) \in B_{1}$ equals to $\left(1-\frac{1}{2} \tau\right)^{2}$. In this case set $\widetilde{W}_{\tau}$ is completely contained in $T_{0}$, i.e.

$$
|U|=\left|\widetilde{W}_{\tau}\right|
$$

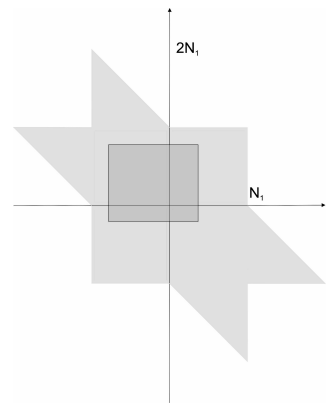

Fig. 4.

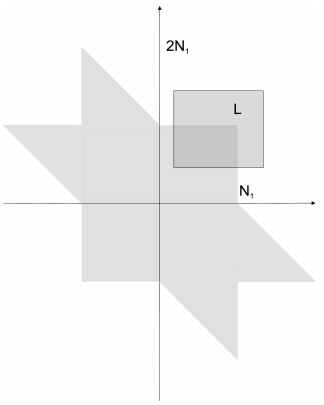

Fig. 5.

2) $\left(n_{1}, n_{2}\right) \in B_{2}=\left\{\left(x N_{1}, y N_{1}\right):|x|>1-\frac{1}{2} \tau O R|y|>1-\frac{1}{2} \tau\right\}$ (see. Fig. 5) Probability of the event $\left(n_{1}, n_{2}\right) \in B_{2}$ equals to $\left(1-\left(1-\frac{1}{2} \tau\right)^{2}\right)$. In this case

$$
|U| \geq \frac{\left|\widetilde{W}_{\tau}\right|}{4} .
$$

Then we find the estimates of the expectation of $Z_{N^{\prime}}$ :

$$
\begin{aligned}
\mathbf{M}\left(Z_{N^{\prime}}\right)= & \left(1-\frac{1}{2} \tau\right)^{2} \mathbf{M}\left(Z_{N^{\prime}} \mid\left(n_{1}, n_{2}\right) \in B_{1}\right) \\
& +\left(1-\left(1-\frac{1}{2} \tau\right)^{2}\right) \mathbf{M}\left(Z_{N^{\prime}} \mid\left(n_{1}, n_{2}\right) \in B_{2}\right)+O\left(N^{\prime \frac{1}{4}}\right) \\
\leq & \left(1-\frac{1}{2} \tau\right)^{2} \sqrt{\frac{\pi N}{4}}+\left(1-\left(1-\frac{1}{2} \tau\right)^{2}\right) \sqrt{\pi N}+O\left(N^{\prime \frac{1}{4}}\right) \\
= & \left(1-\tau+\frac{1}{4} \tau^{2}+2\left(\tau-\frac{1}{4} \tau^{2}\right)\right) \sqrt{\frac{\pi N}{4}}+O\left(N^{\prime \frac{1}{4}}\right) \\
= & \left(1+\tau-\frac{1}{4} \tau^{2}\right) \sqrt{\frac{\pi N}{4}}+O\left(N^{\prime \frac{1}{4}}\right)
\end{aligned}
$$




$$
\begin{gathered}
=\left(1-\frac{1}{2} k\right)^{2} \sqrt{\frac{\pi}{2} \cdot \frac{k^{2} N^{2}}{8} \cdot \frac{4}{k^{2} N}}+\left(1-\left(1-\frac{1}{2} k\right)^{2}\right) . \\
\cdot \sqrt{\frac{\pi}{2} \cdot \frac{k^{2} N^{2}}{8} \cdot \frac{4 \cdot 4}{k^{2} N}}+O\left(N^{\prime \frac{1}{4}}\right) \\
=\left(1-\frac{1}{2} k\right)^{2} \sqrt{\frac{\pi N}{4}}+\left(k-\frac{1}{4} k^{2}\right) \sqrt{\pi N}+O\left(N^{\prime \frac{1}{4}}\right) \\
=\left(1-k+\frac{1}{4} k^{2}+2\left(k-\frac{1}{4} k^{2}\right)\right) \sqrt{\frac{\pi N}{4}}+O\left(N^{\prime \frac{1}{4}}\right) \\
=\left(1+k-\frac{1}{4} k^{2}\right) \sqrt{\frac{\pi N}{4}}+O\left(N^{\prime \frac{1}{4}}\right) .
\end{gathered}
$$

Letting $\tau \rightarrow 0$, we obtain the statement of the theorem.

Theorem is proved.

Theorem 3. Let $G$ be a subgroup of prime order $n$ of the elliptic curve $E$ defined over a finite field $G F(p)$ by equation $y^{2}=x^{3}+A x, p \equiv 1(\bmod 4)$; $\varphi$ be an automorphism of group $G, \varphi(x, y)=(-x, \alpha y)$, where $\alpha$ is the element of order 4 modulo $p ; \lambda$ is the root of the equation $\lambda^{2} \equiv-1(\bmod n)$ such that $\varphi(x, y)=\lambda(x, y)$. Then for any $\varepsilon>0$ there exists an algorithm for the solution of the two-dimensional discrete logarithm problem in the group $G$ with average complexity $(1+\varepsilon) \sqrt{\frac{\pi N}{4}}+O\left(N^{\frac{1}{4}}\right)$ group operations (for $N_{1}=N_{2}, P_{2}=\varphi\left(P_{1}\right)$ and random equiprobable choice of $\left.\left(n_{1}, n_{2}\right)\right)$, where $N=4 N_{1} N_{2}, N \rightarrow \infty$.

Proof. Define «wild» and «tame» sets as it was done in the proof of theorem 2. The shape of the set $T_{0}$ differs from theorem 2 case (see Fig. 2), but the formula remains valid:

$$
A_{N^{\prime}}=2 \cdot \frac{1}{2} \cdot \frac{1}{2} \cdot \frac{4}{N} \cdot \frac{1}{\left|\widetilde{W}_{\tau}\right|} \cdot \sum_{j=1}^{6} j \cdot\left|U_{j}\right|=\frac{2|U|}{N\left|\widetilde{W}_{\tau}\right|}
$$

Subsequent evaluations remain valid too.

Theorem 4. Let $G$ be a subgroup of prime order $n$ of the elliptic curve $E$ defined over a finite field $G F(p)$ by equation $y^{2}=x^{3}+A x+B, p>3 ; \varphi$ is the automorphism of group $G, \varphi(x, y)=(x,-y)$. Then for any $\varepsilon>0$ there exists an algorithm of finding the solution of the two-dimensional discrete logarithm problem in the group $G$ with average complexity $(1+\varepsilon) \sqrt{\frac{\pi N}{2}}+O\left(N^{\frac{1}{4}}\right)$ group operations (for $N_{1}=N_{2}, P_{2}=\varphi\left(P_{1}\right)$ and random equiprobable choice of $\left(n_{1}, n_{2}\right)$ ), where $N=4 N_{1} N_{2}, N \rightarrow \infty$. 
Proof. Define «wild» and «tame» sets as it was done in the proof of theorem 2. Then

$$
A_{N^{\prime}}=2 \cdot \frac{1}{2} \cdot \frac{1}{2} \cdot \frac{2}{N} \cdot \frac{1}{\left|\widetilde{W}_{\tau}\right|} \cdot \sum_{j=1}^{2} j \cdot\left|U_{j}\right|=\frac{|U|}{N\left|\widetilde{W}_{\tau}\right|} .
$$

I.e. $A_{N^{\prime}}$ is twice as much as in the theorem 2 case. Other estimates are valid for this case, however, we get a coefficient $\sqrt{2} \cdot \sqrt{\frac{\pi N}{4}}$.

\section{Conclusion}

Let $E$ be one of the elliptic curves listed above. Then, according to the paper [6], there exists an efficient algorithm of decomposition

$$
k P=k_{1}+k_{2} \varphi(P)
$$

for any point $k P$ of order $n$, where $\varphi$ is an efficient automorphism and $k_{1}, k_{2} \leq$ $C_{G L V} \sqrt{n}$. Therefore, in each of the three cases listed above, the discrete logarithm problem may be reduced to two-dimensional one. Then we obtain

$$
N=4 N_{1} N_{2} \leq 4 C_{G L V}^{2}|G|
$$

In the general case the most effective algorithm of solving discrete logarithm problem is the Pollard algorithm, which may also be accelerated by using endomorphisms. So in the case of an endomorphism of order $q$ the average complexity of the algorithm equals to $\sqrt{\frac{\pi|G|}{2 q}}$ group operations $[6,7]$.

Consider the case of the curve given by equation $y^{2}=x^{3}+A x, p \equiv 1 \bmod 4$, and compare the average complexities of solving the discrete logarithm problem by Gaudry-Schost algorithm and by Pollard algorithm:

$$
\begin{gathered}
(1+\varepsilon) \sqrt{\frac{\pi N}{4}}<\sqrt{\frac{\pi|G|}{2 \cdot 6}}, \\
(1+\varepsilon) \sqrt{\frac{\pi}{4}} \cdot 2 C_{G L V} \sqrt{|G|}<\sqrt{\frac{\pi|G|}{12}} .
\end{gathered}
$$

Thus, when

$$
C_{G L V}<\frac{1}{2 \sqrt{3}(1+\varepsilon)},
$$


theoretical estimates of the average number of group operations of Gaudry-Schost algorithm is smaller.

Similarly, for the case of a curve $y^{2}=x^{3}+B, p \equiv 1(\bmod 3)$,

$$
C_{G L V}<\frac{1}{2 \sqrt{2}(1+\varepsilon)}
$$

and for the case $y^{2}=x^{3}+A x+B, p>3$,

$$
C_{G L V}<\frac{1}{2 \sqrt{2}(1+\varepsilon)} .
$$

\section{References}

[1] Galbraith S.D., Holmes M., "A non-uniform birthday problem with applications to discrete logarithms", Discrete Applied Mathematics, 160:10-11 (2012), 1547-1560, eprint.iacr.org/2010/616.

[2] Galbraith S.D., Ruprai R.S., "An improvement to the Gaudry-Schost algorithm for multidimensional discrete logarithm problems", Cryptography and Coding,, 12th IMA International Conference, Lect. Notes Comput. Sci., 5921, ed. Parker M.G., Springer, 2009, 368-382.

[3] Galbraith S.D., Ruprai R.S., "Using equivalence classes to accelerate solving the discrete logarithm problem in a short interval", Public Key Cryptography $P K C$ 2010, Lect. Notes Comput. Sci., 6056, Springer, 2010, 368-383, eprint.iacr.org/2010/615.

[4] Gaudry P., Schost E., "A low-memory parallel version of Matsuo, Chao and Tsujii's algorithm", Proceedings of Algorithm Number Theory Symposium - ANTS VI, Lect. Notes Comput. Sci., 3076, Springer-Verlag, 2004, 208-222.

[5] Liu W., Improved algorithms for the 2-dimensional discrete logarithm problem with equivalence classes, MSc Thesis, University of Auckland (2010), http://www.math.auckland.ac.nz/ sgal018/Wei-Liu-MSc.pdf.

[6] Wiener M.J., Zuccherato R.J., "Faster attacks on elliptic curve cryptosystems", Lect. Notes Comput. Sci., 1556, 1999, 190-200.

[6] Nikolaev M.V., Matyukhin D.V., "On the complexity of two-dimensional discrete logarithm problem in a finite cyclic group with effective automorphism of order 6", Discrete Math. Appl., 23:3-4, 313-325.

[6] Gallant R., Lambert R., Vanstone S., "Faster point multiplication on elliptic curves with efficient endomorphisms", CRYPTO '01 Proc. 21st Ann. Int. Crypt. Conf. Advances in Cryptology, Lect. Notes Comput. Sci., 2139, ed. Kilian J., 190-200.

[7] Duursma I.M., Gaudry P., Morain F., "Speeding up the discrete log computation on curves with automorphisms", ASIACRYPT'99, Lect. Notes Comput. Sci., 1716, eds. K.-Y. Lam, E. Okamoto, C. Xing, Springer, Heidelberg, 1999, 103-121. 